\title{
EFEITO DE MOLÉCULAS AUTO-ORGANIZÁVEIS NA RESISTÊNCIA À CORROSÃO DA LIGA AA2024-T3
}

\author{
Solange de Souza' \\ Daniel Sierra Yoshikawa ${ }^{2}$ \\ Sérgio Luiz de Assis $^{3}$ \\ Wagner Izaltino Alves dos Santos ${ }^{4}$ \\ Isolda Costa ${ }^{5}$
}

\begin{abstract}
Resumo
Revestimentos baseados em moléculas auto-organizáveis (SAM) vêm sendo estudados como possíveis substitutos aos revestimentos de conversão de cromato para proteção contra corrosão em ligas de alumínio. Neste trabalho é investigada a influência de moléculas auto-organizáveis, do grupo dos alcanos difosfonatos, na resistência à corrosão da liga AA2024-T3 utilizando as técnicas de espectroscopia de impedância eletroquímica, polarização potenciodinâmica, ensaios acelerados de corrosão e microscopia eletrônica de varredura. Os resultados mostram que o tratamento da superfície da liga AA2024-T3 com SAM causou o ataque corrosivo e diminuiu sua resistência à corrosão. $O$ tratamento da superfície com solução à base de cromo $(\mathrm{VI})$ também causou ataque na liga e também diminuiu a sua resistência à corrosão. $O$ tratamento que resulta em melhor resistência à corrosão da liga consiste no crescimento e selagem do óxido de alumínio.
\end{abstract}

Palavras-chave: Moléculas auto-organizáveis; AA2024-T3; Cromatização.

\section{EFFECT OF SELF-ASSEMBLING MOLECULES ON THE CORROSION RESISTANCE OF THE AA2024-T3 ALLOY}

\begin{abstract}
Coatings based on self-assembling molecules (SAM) have been studied as possible substitutes to the chromate conversion coatings for corrosion protection of aluminium alloys. In this work, the influence of alkane diphosphonates self-assembling molecules on the corrosion resistance of the AA2024-T3 aluminium alloy is investigated. The corrosion investigation is carried out by electrochemical impedance spectroscopy, potentiodynamic polarization techniques, accelerated corrosion experiments and scanning electron microscopy technique. Results show that the surface treatment with self-assembling molecules decreased the corrosion resistance of the alloy due to the corrosive attack of the SAM solution to the alloy surface. The solution used for passivation with chromium (VI) also caused the attack of the alloy surface, leading to slight decreases in the corrosion resistance. The surface treatment that produced the best corrosion resistance is associated with the growth and sealing of the surface oxide.
\end{abstract}

Key words: Self-assembling molecules; AA2024-T3; Chromatizing.

\section{INTRODUÇÃO}

Tratamento de superfícies metálicas com camadas de conversão de cromato são processos amplamente utilizados em ligas de alumínio pois, além de aumentarem a sua resistência à corrosão, promovem a aderência de revestimentos orgânicos. Entretanto, o caráter tóxico e poluente do cromo hexavalente, aliado às restrições ambientais, têm incentivado a busca por novos tratamentos que sejam ambientalmente amigáveis e eficientes na proteção à corrosão.(1) Tratamentos de superfícies com filmes de polímeros condutores de eletricidade, por exemplo, polianilina, ${ }^{(2)}$ camadas de conversão de óxidos e hidróxidos de terras raras, principalmente de compostos de cério,(1) deposição de monocamadas auto-organizadas produzidas com moléculas orgânicas de alcano difosfonato, ${ }^{(3)}$ entre outros, ${ }^{(4)}$ estão entre as alternativas que têm sido avaliadas.

'Pós-doutoranda (IPEN/CNEN - CCTM). Av. Prof. Lineu Prestes, 2242, Cidade Universitária, CEP 05508-000, São Paulo, SP, Brasil. E-mail: solangesouza@gmail.com ${ }^{2}$ Mestrando (IPEN/CNEN - CCTM). Av. Prof. Lineu Prestes, 2242, Cidade Universitária, CEP 05508-000, São Paulo, SP, Brasil. E-mail: zina.usp@gmail.com ${ }_{3}^{3}$ Pesquisador (IPEN/CNEN - CCTM). Av. Prof. Lineu Prestes, 2242, Cidade Universitária, CEP 05508-000, São Paulo, SP, Brasil. E-mail: slassis@ipen.br ${ }^{4}$ Mestrando (IPEN/CNEN - CCTM). Av. Prof. Lineu Prestes, 2242, Cidade Universitária, CEP 05508-000, São Paulo, SP, Brasil. E-mail: wagner_quimica@hotmail.com ${ }_{5}^{5}$ Pesquisadora (IPEN/CNEN - CCTM). Av. Prof. Lineu Prestes, 2242, Cidade Universitária, CEP 05508-000, São Paulo, SP, Brasil. E-mail: icosta@ipen.br 
O processo de formação de monocamadas com alto grau de organização estrutural ocorre espontaneamente sobre a superfície de um substrato metálico por adsorção das moléculas surfactantes em solução. ${ }^{(5)}$ Existem vários tipos de moléculas que se enquadram nessa classe, sendo cada molécula específica para um ou mais tipos de substrato. ${ }^{(6)}$ Como exemplo, as moléculas orgânicas à base de difosfonato são específicas para a superfície de óxido de alumínio. ${ }^{(7,8)}$

A formação de camadas auto-organizáveis à base de difosfonato pode ser dividida em duas etapas: $(\mathrm{I})$ a rápida adsorção ao substrato e (2) a lenta organização da camada, determinada por interação intermolecular de Van der Waals entre os alcanos. ${ }^{(6)}$ As moléculas orgânicas à base de difosfonato possuem a vantagem em gerarem uma forte interação entre o metal, ou seu óxido, e a camada de tinta, sendo que, quanto mais compacta for a camada auto-organizada, maior será $\circ$ poder de inibição da reação de redução do oxigênio e maior a estabilidade frente aos produtos gerados por essa reação. ${ }^{(6)}$

Neste trabalho, o uso de moléculas auto-organizáveis como alternativa ao tratamento de cromatização, para prevenir a corrosão da liga AA2024-T3, é investigado por meio das técnicas de espectroscopia de impedância eletroquímica (EIE), polarização potenciodinâmica anódica, ensaio acelerado de corrosão (névoa salina) e de microscopia eletrônica de varredura (MEV).

\section{MATERIAIS E MÉTODOS}

Amostras da liga AA2024-T3 com dimensões de $20 \mathrm{~mm} \times 20 \mathrm{~mm}$ e $2 \mathrm{~mm}$ de espessura, cuja composição química, obtida por Espectrometria de Emissão Óptica com Plasma Induzido (ICP-OES) é apresentada na Tabela I, foram extraídas de uma mesma chapa de origem comercial. Essas amostras foram jateadas com microesferas de vidro com granulometria variando de I 50 a $250 \mu \mathrm{m}$, o que permitiu obter um acabamento Sa3, conforme indicado na norma SIS 055900.(9) $\mathrm{Na}$ sequência, as amostras foram enxaguadas com água deionizada, desengraxadas com etanol,

Tabela I. Composição química (\% m) da liga AA2024-T3

\begin{tabular}{cccrrrr}
\hline $\mathbf{F e}$ & $\mathbf{C u}$ & $\mathbf{M g}$ & \multicolumn{1}{c}{ Mn } & \multicolumn{1}{c}{ Si } & \multicolumn{1}{c}{$\mathbf{Z n}$} & Al \\
\hline 0,163 & 4,060 & 1,771 & 0,626 & 0,106 & 0,019 & Bal. \\
$\pm 0,003$ & $\pm 0,001$ & $\pm 0,011$ & $\pm 0,003$ & $\pm 0,001$ & $\pm 0,001$ & \\
\hline
\end{tabular}

de origem comercial, em banho ultrassônico por cinco minutos e secadas com ar levemente aquecido e, posteriormente, submetidas aos tratamentos detalhados na Tabela 2. As amostras tratadas segundo os procedimentos indicados por OX (crescimento de óxido), SAM (Self-Assembled Monolayer), OS (óxido + Self-Assembled Monolayer) e $\mathrm{Cr}(\mathrm{VI})$ (camada de conversão de cromo), foram previamente submetidas ao tratamento $S R$ (sem revestimento).

Foram realizados ensaios eletroquímicos em solução 0,5 mol.L-1 de $\mathrm{Na}_{2} \mathrm{SO}_{4}$, naturalmente aerada, à temperatura ambiente, com pH ajustado para 4,0 com solução tampão de biftalato de potássio e hidróxido de sódio. Os eletrodos de trabalho, identificados como SR, OX, SAM, OS e $\mathrm{Cr}(\mathrm{VI})$, foram imersos na solução de ensaio e, no terceiro dia de imersão, foram submetidos aos ensaios de EIE seguidos por polarização anódica. Utilizou-se um arranjo experimental de três eletrodos, com fio de platina como contraeletrodo e eletrodo de referência de calomelano saturado em $\mathrm{KCl}(\mathrm{ECS})$. Os diagramas de impedância foram obtidos no potencial a circuito aberto (PCA), na faixa de $100 \mathrm{kHz}$ a $100 \mathrm{mHz}$, com uma amplitude de perturbação de $10 \mathrm{mV}$ (rms) e com uma taxa de aquisição de dados de 10 pontos por década, utilizando um analisador de respostas em frequências, Solartron SI-1260, acoplado ao potenciostato Solartron SI I287A. Foram obtidas curvas de polarização anódica do PCA até $2,5 \mathrm{~V}$, com taxa de varredura de $1 \mathrm{mV} \cdot \mathrm{s}^{-1}$. Foram realizados ensaios de névoa salina durante $168 \mathrm{~h}$, segundo a norma ASTM BII7. Foram realizados ensaios pela técnica de microscopia eletrônica de varredura (MEV) utilizando-se de um microscópio eletrônico de varredura Phillips, modelo XL 30 equipado com um analisador por energia dispersiva de raios $X$ (EDAX). As amostras observadas por MEV foram polidas com pasta de diamante de granulometria de $\mathrm{I}, 0 \mu \mathrm{m}$.

Tabela 2. Tratamentos realizados nos corpos-de-prova da liga AA2024-T3

\begin{tabular}{|c|c|}
\hline Tratamento & Descrição \\
\hline SR & $\begin{array}{l}\text { Ia etapa: Desengraxe em solução de baixa alcalinidade, } \mathrm{pH} 8,5-9,5 . \\
2^{\mathrm{a}} \text { etapa: Desengraxe em solução de alta alcalinidade, } \mathrm{pH} \text { I3,9. } \\
3^{\mathrm{a}} \text { etapa: Desoxidação - Entre cada etapa, as amostras foram enxaguadas com água deionizada. O tempo de imersão em cada } \\
\text { etapa e a temperatura foram } 3 \text { min e }(40 \pm 2)^{\circ} \mathrm{C} \text {, respectivamente. }\end{array}$ \\
\hline OX & SR seguido por imersão durante 30 min em água em ebulição. \\
\hline SAM & $\begin{array}{l}\text { SR seguido por imersão durante } 3 \mathrm{~h} \text { em solução com 90ppm de alcano difosfonato }\left[\mathrm{PO}(\mathrm{OH})_{2}\left(\mathrm{CH}_{2}\right)_{\mathrm{n}} \mathrm{PO}(\mathrm{OH})_{2}\right] \text { da Chemetall } \\
10 \leq \mathrm{n} \leq 12 \text {, } \mathrm{a}(30 \pm 2)^{\circ} \mathrm{C}, \mathrm{pH} 3,5 \text {. }\end{array}$ \\
\hline OS & OX seguido por imersão durante $3 \mathrm{~h}$ em solução com 90 ppm de alcano difosfonato, a $(30 \pm 2)^{\circ} \mathrm{C}$. \\
\hline $\mathrm{Cr}(\mathrm{VI})$ & SR seguido por imersão durante 3 min em solução passivante de cromo hexavalente, a $(40 \pm 2)^{\circ} \mathrm{C}$ e pH 3,7 . \\
\hline
\end{tabular}




\section{RESULTADOS}

Nas Figuras la e Ib são apresentados, respectivamente, os diagramas de Nyquist e de ângulo de fase de Bode para eletrodos com os tratamentos $\mathrm{SR}, \mathrm{Cr}(\mathrm{VI})$ e SAM obtidos após três dias de imersão na solução de ensaio. Nos diagramas de Nyquist observamse arcos capacitivos, sugerindo a interação de várias constantes de tempo e maior impedância para o tratamento SR. Na faixa de média frequência (MF), $10 \mathrm{~Hz}$ a I kHz, as amostras com tratamentos $\mathrm{Cr}(\mathrm{VI})$ e SR apresentam picos relativamente largos com ângulo de fase em torno de $65^{\circ}$ e $70^{\circ}$, respectivamente, enquanto a amostra com tratamento SAM apresenta, na frequência de $10 \mathrm{~Hz}$, um pico estreito e bem definido, com ângulo de fase em torno de $80^{\circ}$. Esses resultados sugerem ataque da superfície da liga pela solução de
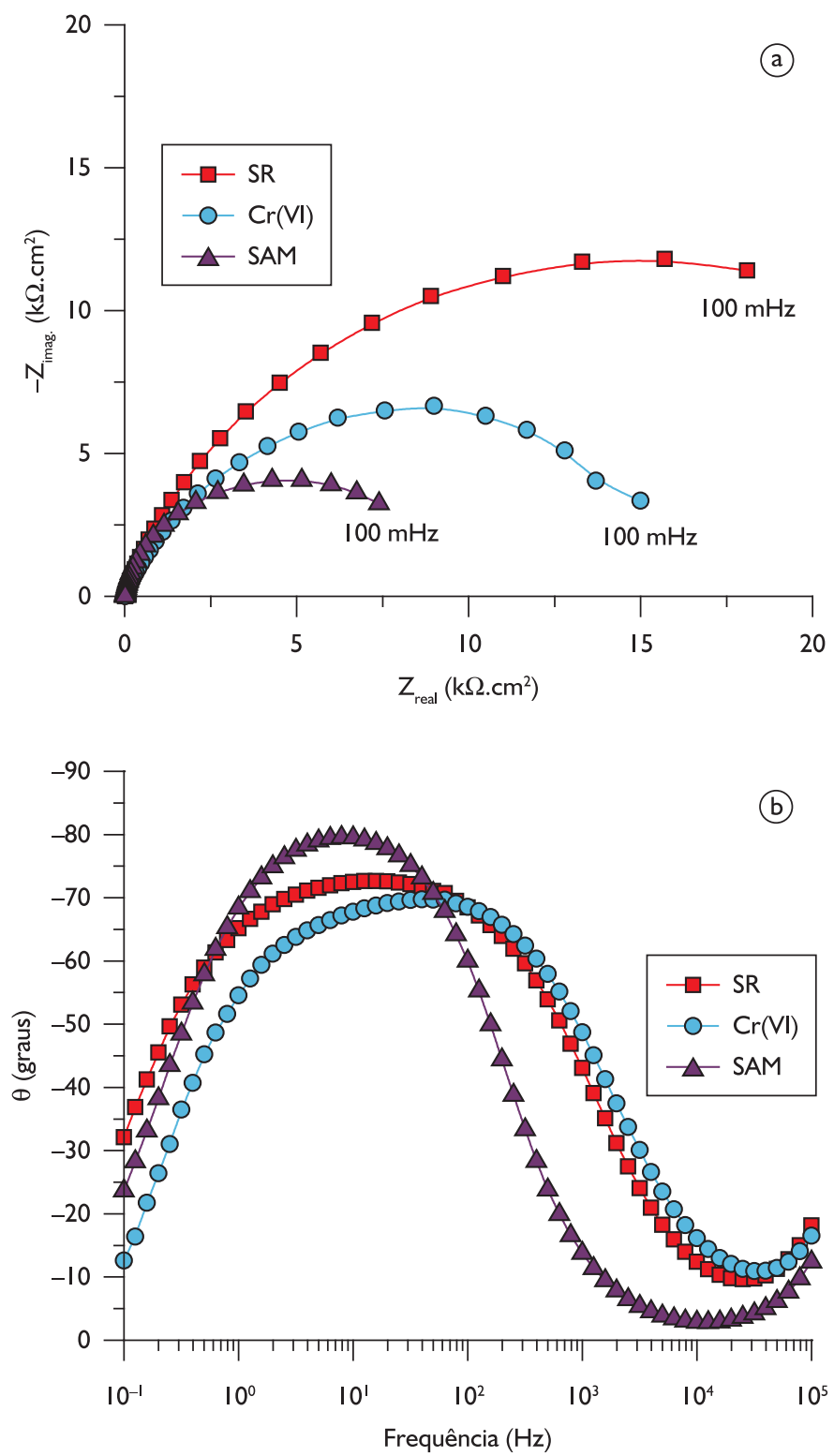

Figura I. Diagramas de: (a) Nyquist e (b) ângulo de fase da liga AA2024-T3 com os tratamentos $\mathrm{SR}, \mathrm{Cr}(\mathrm{VI})$ ou SAM, após três dias de imersão no solução de ensaio.
$\mathrm{Cr}(\mathrm{VI})$ e de SAM. Os tratamentos com $\mathrm{Cr}(\mathrm{VI})$ ou SAM diminuíram a resistência à corrosão da liga em comparação ao tratamento SR. O comportamento mais capacitivo apresentado pela amostra com tratamento $S R$ pode ser justificado pela provável remoção de intermetálicos, do tipo $\mathrm{Al}_{2} \mathrm{Cu}$, uma vez que esse tratamento envolve desengraxe em soluções de baixa e alta alcalinidade, além de uma etapa de desoxidação. A literatura ${ }^{(10,11)}$ reporta que partículas intermetálicas, $\mathrm{Al}_{2} \mathrm{Cu}$, são regiões susceptíveis à corrosão localizada; portanto, acredita-se que a sua remoção, mesma que parcial, tenha contribuído para o melhor desempenho do tratamento SR comparativamente aos tratamentos $\mathrm{Cr}(\mathrm{VI})$ e SAM. As curvas de polarização potenciodinâmicas apresentadas na Figura 2 mostram valores de potencial de corrosão iguais a $-0,15 \mathrm{~V}$, $-0,2$ I V, e - $0,76 \vee(E C S)$, respectivamente para as amostras $\mathrm{SR}, \mathrm{Cr}(\mathrm{VI})$ e SAM. Observa-se, ainda, a partir do potencial de corrosão, um aumento contínuo de densidade de corrente, que alcança valores da ordem de $10^{-4}, 10^{-3}$ e $10^{-5} \mathrm{~A} . \mathrm{cm}^{-2}$, respectivamente, para as amostras $\mathrm{SR}, \mathrm{Cr}(\mathrm{VI})$ e SAM. A partir destes últimos valores de densidade de corrente, nota-se para as amostras SR e $\mathrm{Cr}(\mathrm{VI})$ diminuição na densidade de corrente, que atinge aproximadamente $5 \mu \mathrm{A} \cdot \mathrm{cm}^{-2}$. As amostras com tratamento SAM apresentam potenciais mais ativos em comparação às demais e aumento de corrente seguido pelo estabelecimento de uma densidade de corrente da ordem de $30 \mu \mathrm{A} \cdot \mathrm{cm}^{-2}$, até $\circ$ final da polarização.

Uma vez que as moléculas orgânicas autoorganizáveis usadas têm afinidade pelo óxido de alumínio, ${ }^{(7)}$ investigou-se também $\circ$ efeito do crescimento de óxido (OX) no comportamento eletroquímico da liga (Figuras $3 a$ e $3 b$ ). Nos

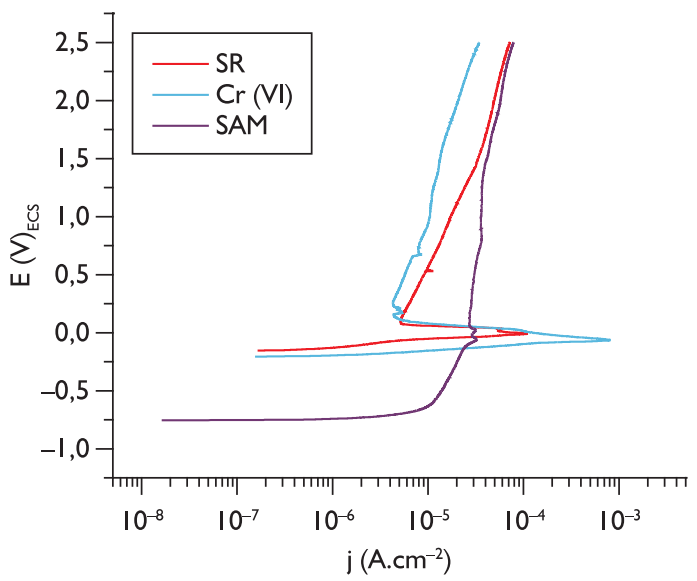

Figura 2. Curvas de polarização potenciodinâmica anódica da liga AA2024-T3 com os tratamentos SR, Cr(VI) e SAM, após três dias de imersão na solução de ensaio. 
diagramas de Nyquist da Figura 3a, ocorre um aumento significativo de impedância associada ao tratamento OX em comparação ao SR. Nos diagramas de ângulo de fase de Bode (Figura 3b), observa-se, na faixa de I $\mathrm{Hz}$ a I kHz, uma nítida separação de duas constantes de tempo para o tratamento OX. O crescimento artificial do óxido de alumínio, promovido por imersão em água fervente, é a causa deste comportamento. Esse tratamento causa tanto o crescimento, como a selagem dos poros da camada de óxido, efeitos que foram altamente benéficos para o aumento da resistência à corrosão da liga de alumínio estudada.

Ligas de alumínio normalmente são submetidas a processos de anodização ou de crescimento artificial de filme de óxido por imersão em água fervente, ou em outros meios com a finalidade de aumentar a resistência à corrosão. ${ }^{(12)}$ Como o objetivo do presente trabalho é avaliar o efeito do tratamento com SAM na
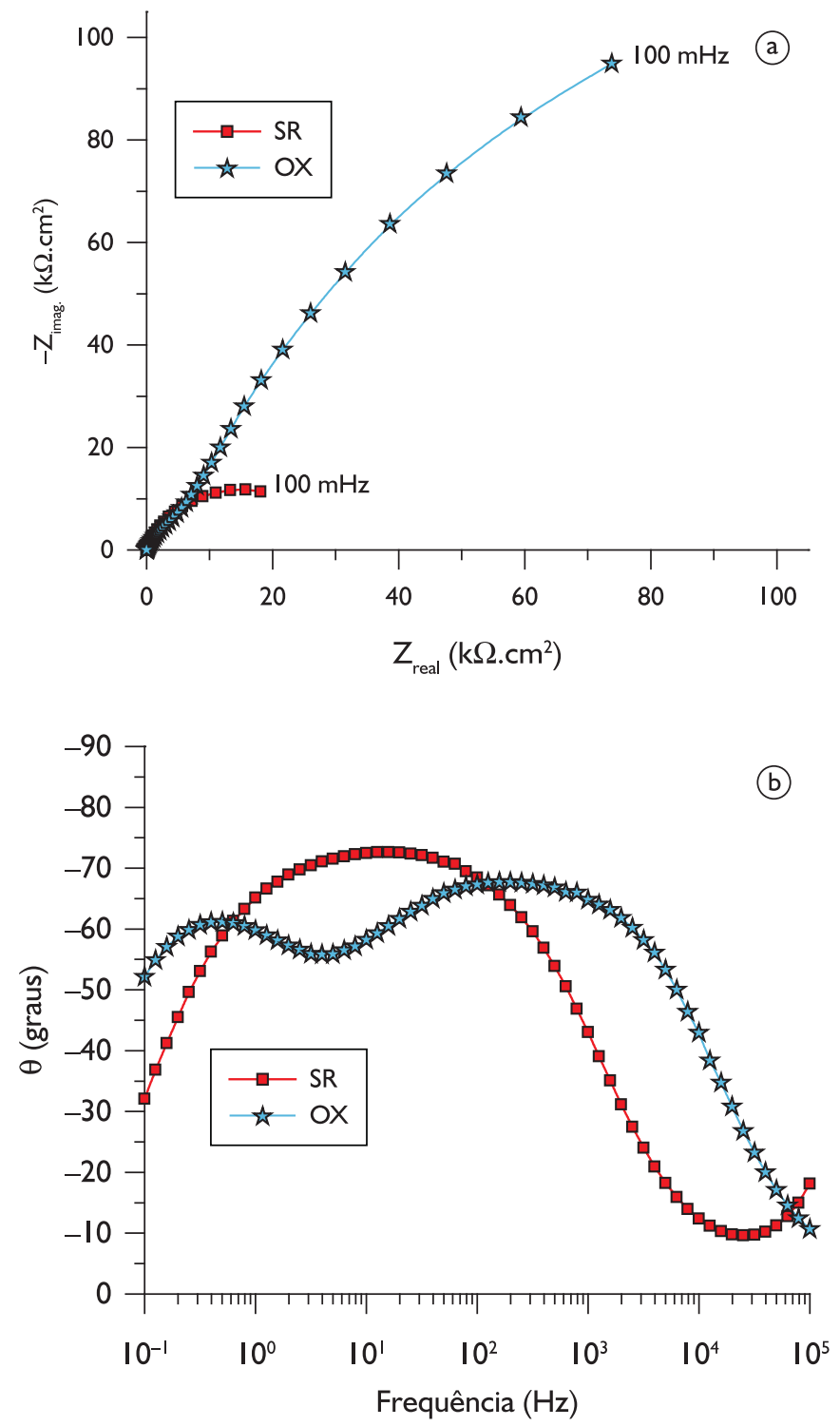

Figura 3. Diagramas de: (a) Nyquist e (b) ângulo de fase de Bode da liga AA2024-T3 com os tratamentos SR ou OX, após três dias de imersão na solução de ensaio. resistência à corrosão da liga, realizou-se também o tratamento SAM em amostras que haviam sido submetidas previamente ao tratamento de crescimento artificial do óxido (OX). Os resultados obtidos com este tratamento, denominado de OS, são mostrados na Figura 4. Nesta figura são comparados os três tratamentos: OS, SR e OX, utilizando diagramas de Nyquist, Figura 4a, e de ângulo de fase de Bode, Figura 4b. Os diagramas de Nyquist, mostram arcos capacitivos achatados e incompletos e maiores valores de impedância para a amostra com tratamento OS em comparação à amostra com tratamento SR. Todavia, o tratamento OX ainda se mostra superior aos demais, fornecendo maiores valores de impedância. $\mathrm{Na}$ faixa de I $\mathrm{Hz}$ a I kHz, os diagramas de ângulo de fase, apresentam nítida separação das duas constantes de tempo para os tratamentos, OX e OS, embora com ângulos de fase aproximadamente $5^{\circ}$ menor para este último. $O$ óxido desenvolvido artificialmente proporciona melhor
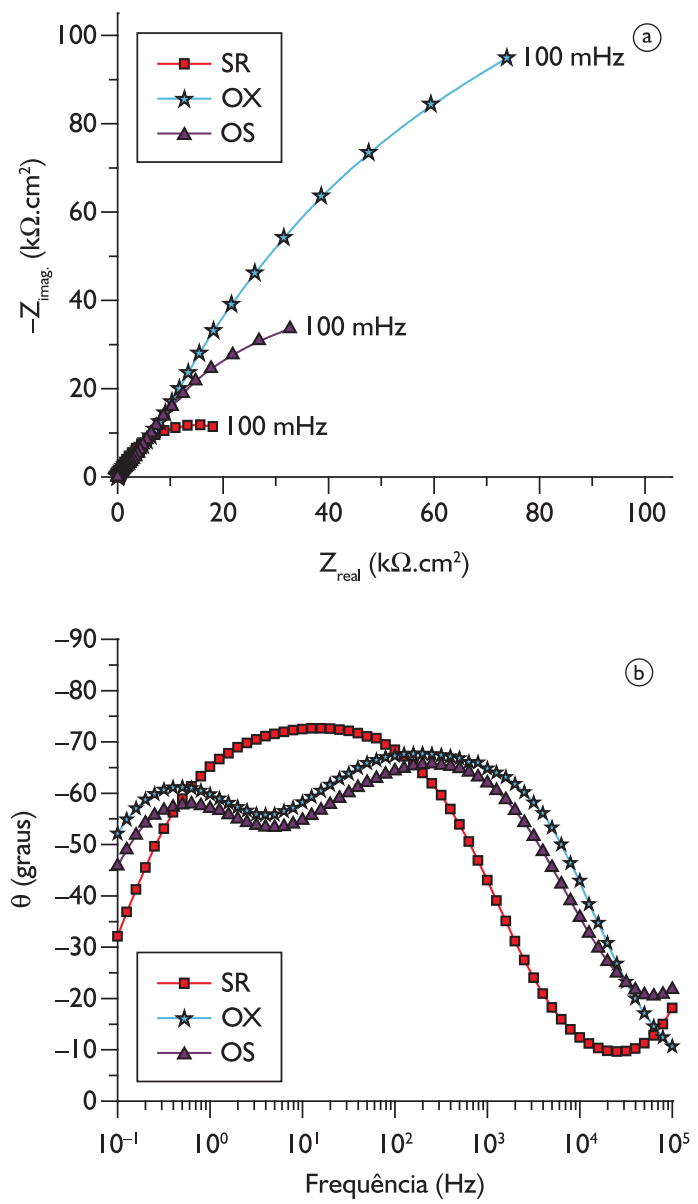

Figura 4. Diagramas de: (a) Nyquist e (b) ângulo de fase de Bode para AA2024-T3 para os tratamentos SR, OX ou OS, com três dias de imersão na solução de ensaio. 
resistência à corrosão. A diminuição de impedância e dos ângulos de fase para as amostras que foram imersas em SAM sugerem o ataque ao óxido crescido artificialmente pela solução com SAM, - que se deve à agressividade desta solução. As curvas de polarização das amostras com os tratamentos SR, OX e OS (Figura 5) mostram o efeito benéfico do óxido formado sobre a superfície da liga. Embora, para sobretensão de 2,5 V(ECS), a densidade de corrente associada ao tratamento OS $\left(\approx 20 \mu \mathrm{A} \cdot \mathrm{cm}^{-2}\right)$ seja menor do que o valor obtido para o tratamento OX $\left(\approx 30 \mu \mathrm{A} \cdot \mathrm{cm}^{-2}\right)$, essa diferença é extremamente pequena para permitir uma classificação hierárquica da resistência à corrosão considerando-se os dois tipos de tratamento de superfície.

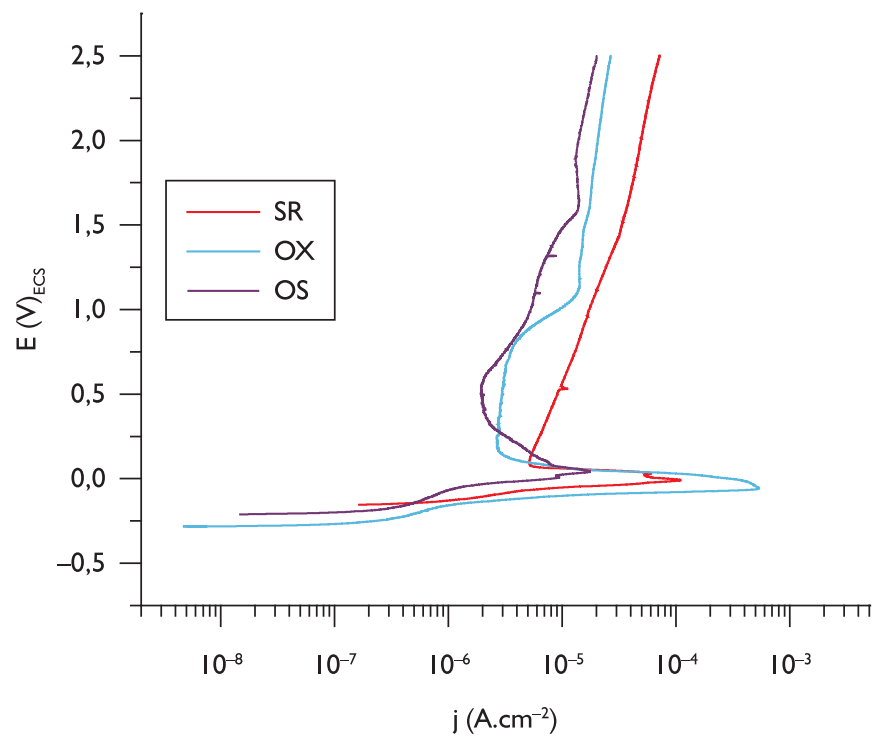

Figura 5. Curvas de polarização potenciodinâmica anódica para AA2024-T3 para os tratamentos SR, OX e OS, após três dias de imersão na solução de ensaio.

\section{DISCUSSÃO}

Os ensaios eletroquímicos revelam diminuição na resistência à corrosão da liga AA2024-T3 quando tratada com SAM. Estes resultados indicam que a superfície da liga foi atacada pela solução ácida de SAM. Esta hipótese é confirmada ao observar a superfície da liga utilizando técnica de MEV. Micrografias de amostras, polida e sem tratamento (Figura 6a), polida + OX (Figura 6b) e polida + OS (Figura 6d), são apresentadas para comparação. $O$ efeito do ataque da solução de SAM é mais pronunciado na superfície da amostra polida do que na polida + OX. Vários pontos de ataque são observados na amostra polida e tratada com SAM, particularmente os pontos associados às regiões de interface entre intermetálicos e a matriz. Em alguns casos, nota-se que $o$ ataque causou a remoção de intermetálicos pequenos. Comportamento similar é indicado na Figura 6(d), porém em menor extensão, mas sempre $o$ ataque está relacionado com intermetálicos. Segundo a literatura, $\mathrm{Al}-\mathrm{Cu}-\mathrm{Mg}^{(1)}$, Al-Cu-Mn-Fe-(Si)(I) e $\mathrm{Al}_{2} \mathrm{Cu}^{(10,11)}$ são as principais partículas intermetálicas presentes na liga AA2024-T3.
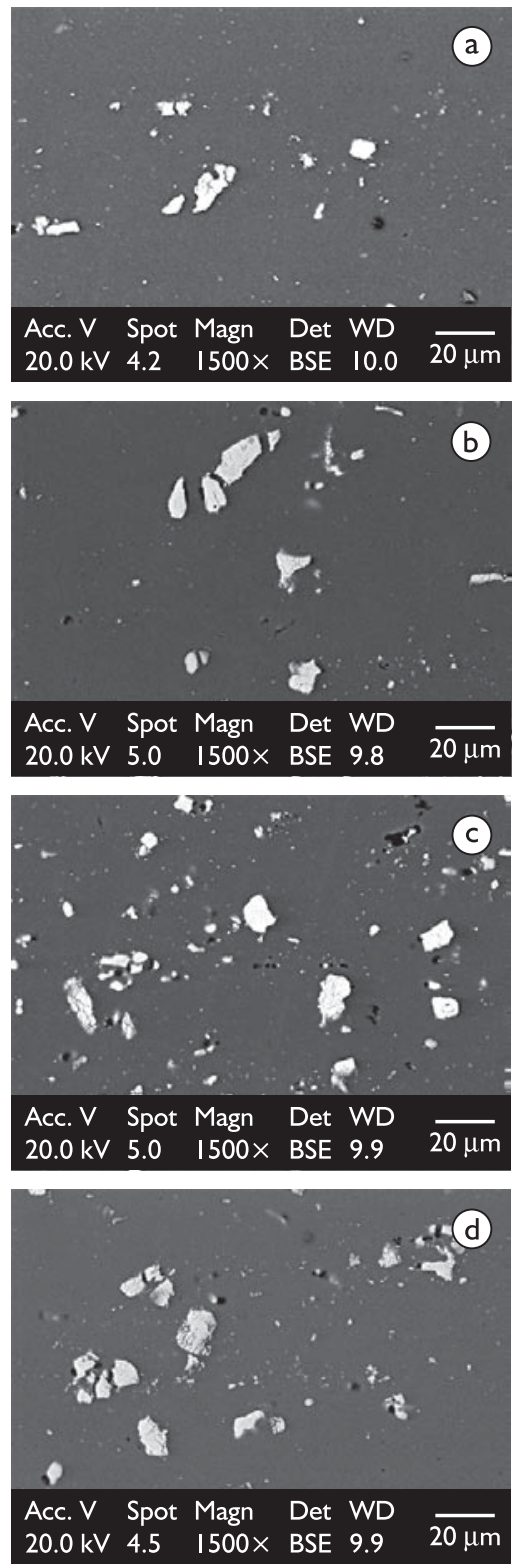

Figura 6. Micrografias da superfície das amostras de liga de alumínio AA2024-T3: (a) polida, (b) polida + OX, (c) polida + SAM e (d) polida + OS.

Pesquisas realizadas anteriormente ${ }^{(13-15)} \mathrm{em}$ nosso laboratório indicaram o efeito benéfico do tratamento com SAM para as ligas de alumínio 1050 , 3003, 5052 e 7475, ao contrário do que é apresentado no presente trabalho. Isso indica que a eficácia do tratamento com SAM é dependente da composição da liga de alumínio, cuja microestrutura está fortemente relacionada a essa composição. As ligas de alumínio, que contêm entre $4 \%$ e $6 \%$ de Cu, são mais suscetíveis à corrosão localizada, necessitando de tratamentos de proteção para a sua utilização em atmosferas de média e alta agressividades. Tais tratamentos podem ser realizados por revestimentos de conversão, como anodização ou cromatização, 
geralmente seguidos por revestimentos orgânicos, ou cladização com alumínio, isto é, revestimento com alumínio comercialmente puro. Os resultados do presente trabalho revelam que AA2024-T3 sem clad é suscetível ao ataque corrosivo da solução de SAM. Ensaios de névoa salina da liga AA2024-T3, nas condições SR, Cr(VI), OX, SAM e OS, indicam a mesma tendência dos ensaios eletroquímicos, observandose maior resistência à corrosão associada com tratamentos OX e OS. Estes dois últimos tratamentos resultaram no crescimento do óxido/ selagem dos poros por imersão em água fervente e promoveram o aumento da resistência à corrosão da liga AA2024-T3 em comparação aos tratamentos com $\mathrm{Cr}(\mathrm{VI})$ ou SAM.

\section{CONCLUSÕES}

O tratamento da liga AA2024-T3 com moléculas auto-organizáveis (SAM) do grupo dos alcanos difosfonato, em solução com pH 3,5 não proporciona proteção contra a corrosão. Na realidade, esse tratamento ocasionou ataque da superfície, particularmente em regiões de interface entre a matriz e intermetálicos, reduzindo a resistência à corrosão da liga AA2024-T3. O desenvolvimento de óxido superficial por imersão em água fervente fornece maior resistência à corrosão do que os tratamentos com $\mathrm{Cr}(\mathrm{VI})$ ou SAM. O tratamento com SAM após crescimento do óxido (OS) provoca, também, um ligeiro ataque ao filme de óxido. Apesar deste ataque, a resistência à corrosão da liga com tratamento OS superior à da liga com tratamento à base de $\mathrm{Cr}(\mathrm{VI})$. Os resultados do presente trabalho permitem concluir que o tratamento SAM não é recomendável para a liga AA2024-T3.

\section{Agradecimentos}

À FAPESP pelos aportes financeiros (Processos: 2004/I2189-I e 2007/55383-0), à Capes e ao CNPq.

\section{REFERÊNCIAS}

I PALOMINO, L.E.M.; AOKI, I.V.; MELO, H.G. Microstructural and electrochemical characterization of Ce conversion layers formed on Al alloy 2024-T3 covered with Cu-rich smut. Electrochimica Acta, v. 5I, n. 28, p. 5943-53, Sep. 2006.

2 DE SOUZA, S. Smart coating based on polyaniline acrylic blend for corrosion protection of different metals. Surface and Coatings Technology, v. 20I, n. I6-17, p. 7574-8I, May 2007.

3 REIS, F.M.; MELO, H.G.; COSTA, I. EIS investigation on AI 5052 alloy surface preparation for self-assembling monolayer. Electrochimica Acta, v. 5 I, n. 8-9, p. I780-8, Jan. 2006.

4 HINTZE, P.E.; CALLE, L.M. Electrochemical properties and corrosion protection of organosilane self-assembled monolayers on aluminium 2024-T3. Electrochimica Acta, v. 5I, n. 8-9, p. I76I-66, Jan., 2006.

5 SCHREIBER, F. Structure and growth of self-assembling monolayers. Progress in Surface Science, v. 65, n. 5-8, p. I5I-256, Dec. 2000.

6 STEWART, K.R. et al. Improved adhesion of thin conformal organic films to metal surface. Reviews of Scientific Instruments, v. 57, n. 7, p. I38I-3, Mar. 1986

7 MAEGE, I. et al. Self-assembling adhesion promoters for corrosion resistant metal polymer interface. Progress In Organic Coatings, v. 34, n. I-4, p. I-I2, July 1998.

8 ULMAN, A. Thin film "organic thin films and surfaces: directions for the nineties". New York: Academic Press, 1995.

9 INTERNATIONAL ORGANIZATION FOR STANDARDIZATION. Norma ISO 850 I - I: I 988. Preparation of steel substrates before application of paints and related products: visual assessment of surface cleanliness. Part I: Rust grades and preparation grades of uncoated steel substrates and of steel substrates after overall removal of previous coatings. Disponível em: http://www.iso.org/iso/iso_catalogue/catalogue_ics/ catalogue_detail_ics.htm?csnumber= I57| I \&ICSI =25\&ICS2=220\&I CS3 $=10$. Acesso em: 20 fev. 2009.

IO OSÓRIO, W.R., et al. The roles of macrosegregation and of dendritic array spacings on the electrochemical behavior of an Al-4.5 wt.\% Cu alloys. Electrochimica Acta, v. 52, n. 9, p. 3265-73, Feb. 2007.

I I OSÓRIO, W.R. et al. Corrosion behavior of hypoeutectic $\mathrm{Al}-\mathrm{Cu}$ alloys in $\mathrm{H}_{2} \mathrm{SO}_{4}$ and $\mathrm{NaCl}$ solutions. Acta Metallurgica Sinica, v. 22, n. 4, p. 24I-6, Aug. 2009.

I2 BALTAT-BAZIA, A. et al. Electrochemical impedance spectroscopy and electron microscopies applied to the structure of anodic oxide layers on pure aluminium. Materials Science Forum, v. I I I I I 2, p. 359-68, 1992.

I 3 IZALTINO, W.A.S. et al. Self Assembling Molecules as corrosion inhibitors for 1050 aluminum alloy. In: EUROCORR 2009, Nice, France. Proceedings... Frankfurt: European Federation of Corrosion, 2009.

I 4 YOSHIKAWA, D.S. et al. Estudo do comportamento de corrosão da liga 5052 tratada com moléculas auto-organizáveis. Tratamento de Superfície, v. I, n. I55, p. 24-9, Maio/Jun. 2009.

I5 DE SOUZA, S. et al. Nanostructured surface pre-treatment based on Self-Assembled Molecules for corrosion protection of Alclad 7475-T76 I aluminium alloy. In: INTERNATIONAL CONFERENCE ON ADVANCED MATERIALS, I I; ENCONTRO DA SBPMAT, 8., 2009, Rio de Janeiro. Rio de Janeiro: Sociedade Brasileira de Pesquisa em Materiais, 2009. I CD-Rom.

Recebido em: 27/10/2009

Aceito em: 23/02/2010 